\title{
Variaciones en la abundancia de peces en sitios con diferente grado de contaminación del sublitoral de Ciudad de La Habana, Cuba
}

\author{
Ivet Hernández Hernández, Consuelo Aguilar Betancourt \& Gaspar González Sansón \\ Centro de Investigaciones Marinas, Universidad de La Habana, Calle 16 e/ 1ra y 3ra. No. 114. Miramar. Ciudad de \\ La Habana. Cuba CP: 11300; marketing@transtur.cu
}

Recibido 29-XI-2008. Corregido 10-IV-2009. Aceptado 13-V-2009.

\begin{abstract}
Abundance variations of fishes from sites with different levels of pollution in the sublittoral sector of Havana City, Cuba. The impact of human activity affects fish populations. We studied the abundance of three selected fish species (Stegastes partitus, Thalassoma bifasciatum and Halichoeres bivittatus) in four sites with different degrees of pollution in Havana City: $30^{\text {th }}$ street and $16^{\text {th }}$ street; Miramar; Red Boy of Havana Bay; and the ending of Almendares's River. The sites were divided into five zones depending on their bottom characteristics. The counts were made with the visual census technique with a $5 \mathrm{~m}^{2}$ quadrat, from June-July 2003 to January 2004. Each time we made nine counts per zone. S. partitus and T. bifasciatum were more abundant in clean water areas, while Halichoeres bivittatus seems to resist the levels of pollution present in the area. Rev. Biol. Trop. 57 (4): 977-992. Epub 2009 December 01.
\end{abstract}

Key words: Stegastes partitus, Halichoeres bivittatus, Thalassoma bifasciatum, fishes, spatial abundance.

El impacto humano sobre los ecosistemas marinos se manifiesta de una forma compleja. El análisis de sus alteraciones físicas o químicas no permite pronosticar la manera en que se afectarán determinados componentes de las comunidades. Por este motivo es muy recomendable investigar de forma directa los cambios en la composición por especies de diferentes asociaciones.

La base para utilizar el monitoreo biológico de los peces con el objetivo de evaluar la degradación ambiental, es que la salud relativa de una comunidad de peces es un indicador sensible de los impactos directos e indirectos sobre el ecosistema acuático en su conjunto (Fausch et al. 1990). Este aspecto fue aclarado por Karr \& Dudley (1981), los cuales definieron la integridad biológica de los ecosistemas acuáticos como la capacidad de sostener y mantener una comunidad de organismos balanceada, integral y adaptada, la cual posee una composición por especies, una diversidad y composición funcional proporcional a la de sus hábitats naturales. Los enfoques más difundidos, para el análisis de las comunidades de peces, incluyen la utilización de especies y grupos de especies indicadoras.

En la zona entre la bahía de La Habana y la calle 30 de Miramar se han desarrollado trabajos que incluyen una caracterización de las poblaciones de corales, gorgonias, esponjas (Herrera \& Alcolado 1983, Alcolado \& Herrera 1987, Herrera \& Martínez-Estalella 1987, Guardia 2000a, 2000b, Guardia \& GonzálezSansón 2000a, 2000b) y peces (Aguilar et al. 1997, 2000, González-Sansón \& Aguilar 2000, en prensa, Aguilar \& González-Sansón 2002). Los resultados de estos estudios pusieron en evidencia la existencia de un gradiente de deterioro de las comunidades bentónicas a lo largo de la costa, relacionado principalmente con el desagüe de la Bahía de La Habana. Esos 
trabajos reportaron que las principales alteraciones en las comunidades de invertebrados se encontraron en las estaciones al Oeste de la Bahía, mientras que en la zona de Miramar los índices ecológicos indicaron que las condiciones de contaminación no eran tan severas.

Por la escasez de trabajos cuantitativos realizados sobre la variación espacial de las poblaciones de peces, el presente trabajo tiene como objetivo determinar la variación espacial y temporal de la abundancia de esas especies en sitios con diferente grado de contaminación. Para ello fueron seleccionadas 3 especies de peces: Stegastes partitus (Poey 1868), Thalassoma bifasciatum (Bloch 1791) y Halichoeres bivittatus (Bloch 1791). Estas reunieron las siguientes características: estar bien representadas en la zona de estudio (fueron las más abundantes), presentar una elevada afinidad con el sustrato y presentar diferentes hábitos de alimentación.

\section{MATERIALES Y MÉTODOS}

El área de estudio es la porción del sublitoral entre 2 y 18 metros de profundidad entre la calle 30 de Miramar y la Boya Roja, que marca la entrada de la Bahía de La Habana. Se ubicaron 4 sitios que se corresponden con (Fig. 1): las

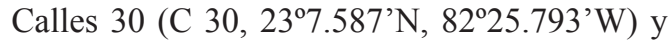
$16\left(\mathrm{C} 16,213^{\circ} 7.919^{\prime} \mathrm{N}, 82^{\circ} 25.179^{\prime} \mathrm{W}\right)$, de Miramar, el lado Este de la desembocadura del río Almendares (Río, 23ㅇ․ $250^{\circ} \mathrm{N}, 82^{\circ} 24.565^{\circ} \mathrm{W}$ ) y el lado Oeste de la entrada de la bahía de La Habana (BR, 23ㅇ․ $864^{\prime} \mathrm{N}, 82^{\circ} 21.576^{\prime} \mathrm{W}$ ).

Los sitios seleccionados presentan diferente grado de afectación por la contaminación. La Bahía de La Habana es una típica bahía de bolsa de las más afectadas del mundo por los residuos industriales, aguas de albañal y petróleo (Ibarra et al. 1983), y su actividad portuaria es de gran peso en la ciudad (Milliar 1997). Las aguas contaminadas de la Bahía soportan desde 1994 la descarga de un total de 56 fuentes contaminantes terrestres y la contaminación química proveniente del transporte marítimo de combustible. Entre las fuentes terrestres se encuentran: las aguas negras de la ciudad; las industrias de procesamiento de azúcar, carne y cuero; de acabado de metales; de generación de vapor y electricidad; así como una refinería de petróleo y una planta de fertilizantes, lo que implica una alta entrada de materia orgánica disuelta y particulada, contaminación por hidrocarburos, aceite y metales pesados. Todo ello actúa en detrimento de la estabilidad biológica del ecosistema (Proyecto Cub-80-001). Por el gran volumen de contaminantes provenientes de la bahía, BR se consideró como un área muy contaminada.

El Río Almendares por su parte, es uno de los más contaminados de Ciudad de La Habana y por tanto, aporta gran cantidad de elementos contaminantes que se vierten al mar. Este Río se encuentra entre los recursos más explotados del Parque Metropolitano, y su gran contaminación se debe a que recibe directamente los vertimientos de las industrias, de la agricultura, de las actividades recreativas y de la población que se asienta en el territorio del Parque (Díaz 1997). El Río drena un área de $402.02 \mathrm{~km}^{2}$ entre las provincias de La Habana y Ciudad de La Habana y presenta grandes problemas de contaminación de origen industrial y doméstico. Por

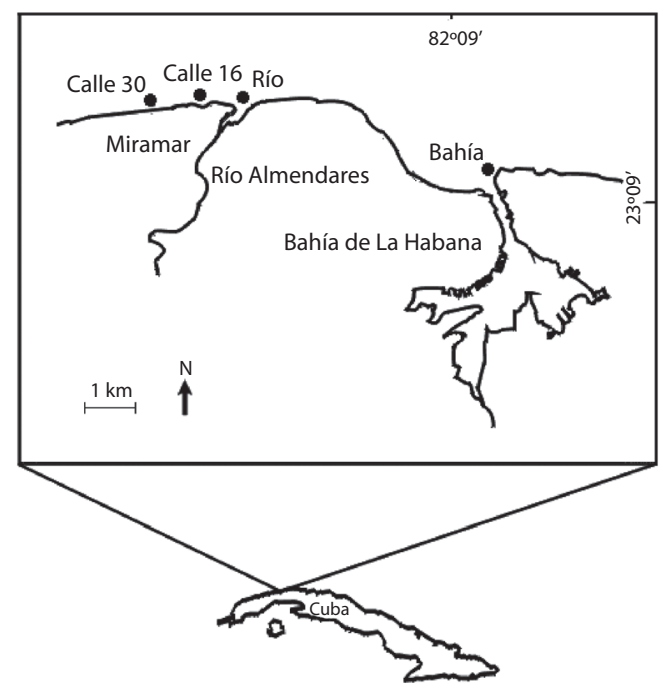

Fig. 1. Zona de estudio.

Fig. 1. Study area. 
las aguas contaminadas provenientes del río, el sitio ubicado en su desembocadura se consideró como medianamente contaminado.

Se asume que los sitios de C 16 y C 30 se pueden considerar como áreas aceptables de referencia debido a que cerca de ellos no existe ninguna fuente notable de contaminación, especialmente C 30 . Además, la existencia de una corriente casi permanente pero de intensidad variable en dirección. Este ayuda a que estos sitios se mantengan limpios (Aguilar \& González-Sansón 2000).

Los 4 sitios de trabajo se dividieron en 5 biotopos según sus características de fondo; estos corren paralelos a la costa y se definen como (Hernández-Hernández et al. 2006):

- Echinometra: Fondo rocoso con numerosas hoquedades que le sirven de refugio a una fauna típica de equinodermos del género Echinometra fundamentalmente (profundidad de $3 \mathrm{~m}$ ).

- Plano Rocoso o Planicie: Comienza a partir de los $3 \mathrm{~m}$ de profundidad y se extiende hasta los $6 \mathrm{~m}$. El fondo es rocoso y plano con zanjas pequeñas donde se agrupan concentraciones altas de peces juveniles.

- Veril Superior: Zona rocosa que colinda inmediatamente con la parte alta de la pendiente del veril. Posee un sustrato irregular y abundante fauna macrobentónica. Abundan esponjas, corales masivos y gorgonáceos. Se encuentra a una profundidad de 6-10m.

- Pendiente: Zona rocosa que se extiende desde los $10 \mathrm{~m}$ hasta los $15 \mathrm{~m}$ de profundidad y se caracteriza por una pendiente abrupta. Presenta una alta complejidad topográfica.

- Veril Inferior: Está formado por una franja de arena que colinda de forma inmediata con la pendiente del veril a una profundidad media de $15 \mathrm{~m}$. El tipo de sustrato determina una flora escasa.

Método de muestreo: Se utilizó como unidad de muestreo, una cuadrícula de 5x5 metros. Con el objetivo de determinar las variaciones en la abundancia, se colocaron 9 unidades de muestreo en cada uno de los 5 biotopos de los 4 sitios. Se obtuvo un total de 45 unidades de muestreo por sitio y se contaron todos los individuos pertenecientes a las especies Stegastes partitus, Halichoeres bivittatus y Thalassoma bifasciatum en los periodos de junio-julio del 2003 (época de lluvia) y enero del 2004 (época de seca). En total, 45 unidades muestrales (UM) por sitio en tres visitas, en cada época. Todas las observaciones se realizaron mediante buceo autónomo. En cada conteo se realizaron mediciones de salinidad. En todos los casos la salinidad resultó ser oceánica por lo que consideramos que a los sitios estudiados no llega la influencia directa de las descargas de agua dulce proveniente del río. Las únicas diferencias apreciables entre los sitios resultan los contaminantes disueltos en el agua y la eutrofización.

En los biotopos de cada sitio se predeterminaron grupos de tallas de $0-3 \mathrm{~cm}, 3-6 \mathrm{~cm}$, $6-10 \mathrm{~cm}$ y más de $10 \mathrm{~cm}$ de longitud y se agruparon a los individuos de cada especie en el grupo correspondiente mediante observación directa in situ empleando una escala graduada. Se consideró que para la especie Stegastes partitus, los juveniles pertenecen al primer grupo de tallas, mientras que los adultos conforman el grupo de 3- $6 \mathrm{~cm}$. Para el resto de las especies se consideraron juveniles a los individuos pertenecientes de $0-6 \mathrm{~cm}$ y adultos a los correspondientes a las longitudes desde 6 hasta los mayores de $10 \mathrm{~cm}$ de longitud.

Para el análisis de los datos de abundancia se utilizó el análisis de varianza, considerando un diseño bifactorial de efectos fijos en el cual los factores son el sitio de muestreo (4 niveles) y la época de muestreo (2 niveles), para cada biotopo por separado. Previo a la realización del análisis se determinó si existía dependencia de la varianza con respecto a la media del número de individuos en los conteos combinados y los datos fueron transformados utilizando como guía el valor de la pendiente de la línea de regresión del logaritmo de la varianza sobre el logaritmo de la media (Elliot 1971). En todos 
los casos la transformación apropiada resultó ser la raíz cuarta de los datos originales. Los análisis de varianza se hicieron sobre los datos transformados. En los casos en los cuales se rechazó la hipótesis nula, se efectuó una prueba de comparaciones múltiples a posteriori utilizando el método de Student-Newman Keuls o prueba SNK (Zar 1996). La representación gráfica de los valores medios de los niveles de los factores o las interacciones para los cuales se encontraron diferencias significativas se basó en los datos sin transformar. El nivel de significación de todas las pruebas fue de 0.05. Para el procesamiento de los resultados se empleó el programa Microsoft Excel para Windows y el programa STATISTICA 5.0.

\section{RESULTADOS}

\section{Variación en la abundancia en sitios con diferente grado de contaminación:}

\section{Stegastes partitus}

La especie Stegastes partitus está presente en todos los sitios con patrones muy similares de abundancia (Fig. 2). Se observan máximos de mayor abundancia en los biotopos más profundos de Veril Superior y Pendiente que son los de mayor complejidad topográfica. La abundancia disminuye algo en el Veril Inferior y más aún en los biotopos de Planicie y Echinometra. Por esta razón en estos dos biotopos no se aplicó una prueba estadística.

Stegaster partitus

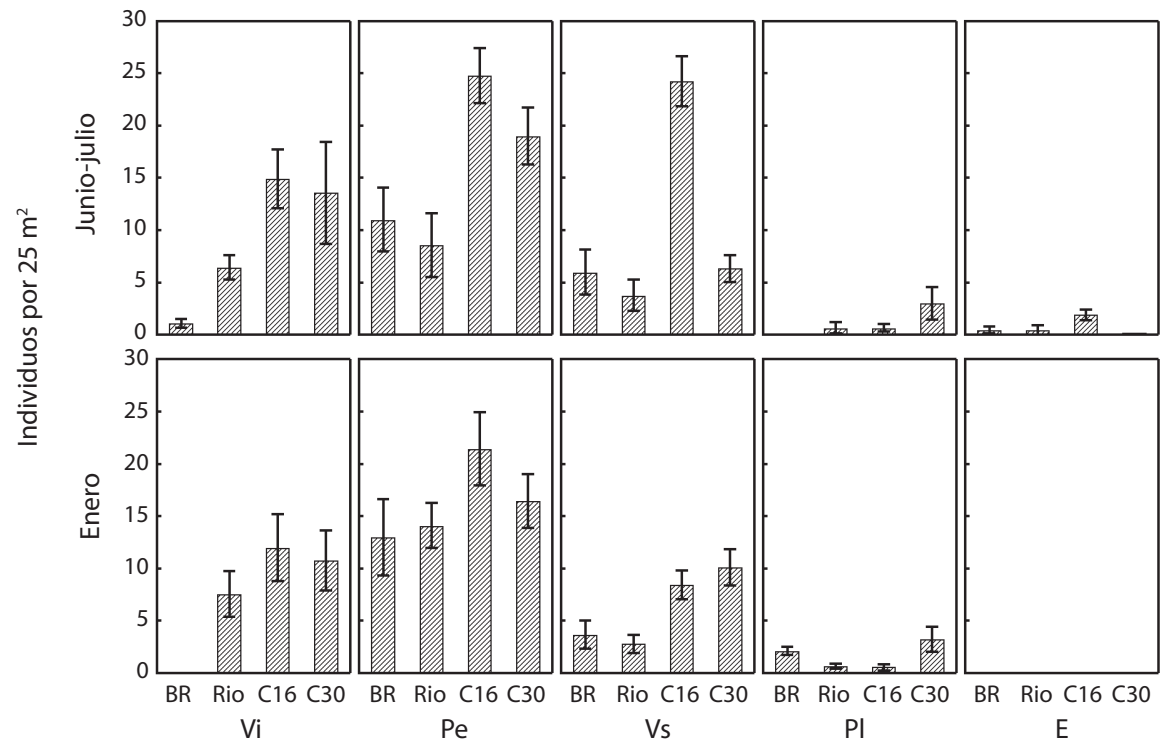

Fig. 2. Abundancia de Stegastes partitus en los distintos biotopos (E: Echinometra; Pl: Plano Rocoso; Vs: Veril Superior; Pe: Pendiente; Vi: Veril para todos los sitios muestreados (BR: Boya Roja; Rio: Almendares; C16: Calle 16; C30: Calle 30). Se representa la media y el error estándar.

Fig. 2. Stegastes partitus abundance at different sites (E: Echinometra; Pl: Rocky Plain; Vs: Superior Wall; Pe: Wall; Vi: Inferior Wall) in sampled sites (BR: Red Buoy; Rio: Almendares river; C16: 16 street; C30: 30 street). Mean and standard error are represented. 
En esta especie resultó notable su escasa abundancia en el Veril Inferior de Boya Roja. Al realizar un análisis de varianza se encontró que Boya Roja fue el único sitio que presentó una media significativamente menor que el resto, los cuales eran semejantes entre sí. Las épocas no eran disímiles y tampoco existía interacción. Aunque la abundancia de esta especie en Boya Roja no es muy elevada pueden encontrarse individuos con mayor frecuencia en la Pendiente, lo cual concuerda con lo que ocurre en el resto de los sitios. En este biotopo la interacción entre sitios y épocas del año resultó significativa. El Río, en lluvia, resultó tener una media significativamente menor que el resto, que eran iguales entre sí. Por último, en el Veril Superior encontramos nuevamente las diferencias entre sitios nada más y esta vez las medias presentes en Boya Roja y Río no diferían entre sí pero ambas eran significativamente menores que las de $\mathrm{C} 30$ y C 16, que no diferían entre ellas (Cuadro 1).

\section{Halichoeres bivittatus}

C 30 y C 16 mostraron una abundancia de Halichoeres bivittatus en el Veril Inferior fundamentalmente. El Río es el sitio en el que los estos peces son más escasos. La mayoría se encuentran en el Veril Inferior. Boya Roja resultó ser el de mayor abundancia y tamaño de estos peces, especialmente en la Planicie y el Veril Superior en lluvia (Fig. 3). El análisis de varianza por biotopos permitió corroborar estas afirmaciones generales (Cuadro 2).

\section{CUADRO 1}

Resultados del análisis de varianza y la prueba SNK por biotopos para Stegastes partitus entre épocas del año y los sitios estudiados

TABLE 1

Stegastes partitus ANOVA and SNK tests results by site between seasons and sites

\begin{tabular}{|c|c|c|c|c|}
\hline Fuente de Variación & Grado de libertad & Cuadrado Medio & $\mathrm{F}$ & $P$ \\
\hline \multicolumn{5}{|l|}{ VERIL INFERIOR } \\
\hline Sitios & 3 & 6.745 & 17.191 & $<0.001$ \\
\hline Epocas & 1 & 1.492 & 3.802 & 0.056 \\
\hline $\mathrm{SxE}$ & 3 & 0.146 & 0.371 & 0.774 \\
\hline Error & 64 & 0.392 & & \\
\hline SNK & BR(b) Rio(a) C16 (a) C30(a) & & & \\
\hline \multicolumn{5}{|l|}{ PENDIENTE } \\
\hline Sitios & 3 & 1.316 & 7.048 & $<0.001$ \\
\hline Epocas & 1 & 0.434 & 2.323 & 0.132 \\
\hline $\mathrm{SxE}$ & 3 & 0.654 & 3.501 & 0.020 \\
\hline Error & 64 & 0.187 & & \\
\hline \multirow[t]{2}{*}{ SNK } & \multirow{2}{*}{\multicolumn{4}{|c|}{$\begin{array}{l}\text { BR-LL(a) BR-S(a) Rio-LL(b) Rio-S(a) } \\
\text { C16-LL(a) C16-S(a) C30-LL(a) C30-S(a) }\end{array}$}} \\
\hline & & & & \\
\hline \multicolumn{5}{|l|}{ VERIL SUPERIOR } \\
\hline Sitios & 3 & 3.478 & 11.242 & $<0.001$ \\
\hline Epocas & 1 & 0.486 & 1.570 & 0.215 \\
\hline $\mathrm{SxE}$ & 3 & 0.660 & 2.134 & 0.105 \\
\hline Error & 64 & 0.309 & & \\
\hline SNK & \multicolumn{4}{|l|}{ BR(b) Rio(b) C16 (a) C30(a) } \\
\hline
\end{tabular}




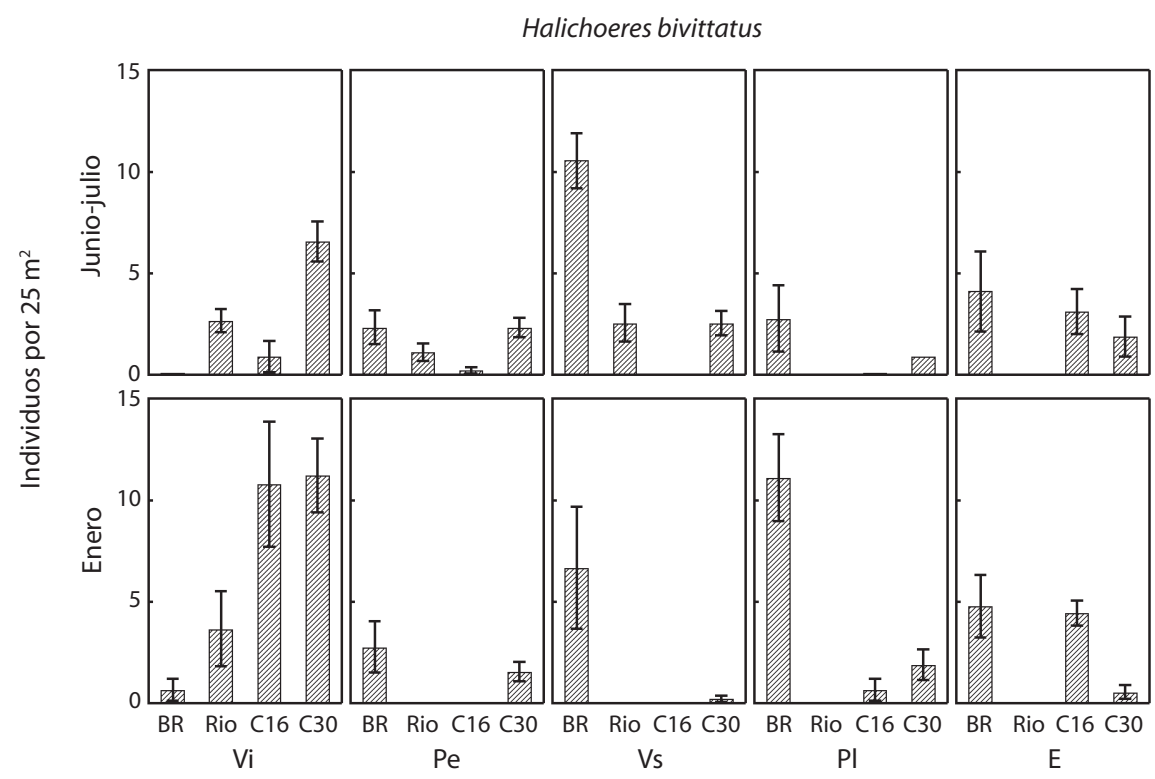

Fig. 3. Abundancia de Halichoeres bivittatus en los distintos biotopos (E: Echinometra; Pl: Plano Rocoso; Vs: Veril Superior; Pe: Pendiente; Vi: Veril Inferior) para todos los sitios muestreados (BR: Boya Roja; Rio: Almendares; C16: Calle 16; C30: Calle 30). Se representa la media y el error estándar.

Fig. 3. Halichoeres bivittatus abundance at different sites (E: Echinometra; P1: Rocky Plain; Vs: Superior Wall; Pe: Wall; Vi: Inferior Wall) in sampled sites (BR: Red Buoy; Rio: Almendares river; C16: 16 street; C30: 30 street). Mean and standard error are represented.

En el Veril Inferior se encontró interacción significativa entre sitios y épocas. La prueba de comparación múltiple no permitió definir grupos de medias similares, aunque se observa claramente que los sitios limpios en ambas épocas tienden a presentar abundancias mayores que los afectados.

Para la Pendiente fueron disímiles entre sí los sitios y las épocas, siendo lluvia la que presentaba los valores más elevados de las medias de abundancia. C 30 y Boya Roja no diferían entre ellos pero sí con Río y C 16, los cuales resultaron ser similares.

En el Veril Superior resultó significativa la interacción. Se vio que Boya Roja tenía la media de abundancia significativamente superior al resto en época de lluvia. Lo seguían en abundancia Boya Roja en seca, C 30 en lluvia y Río en lluvia los cuales forman otro grupo de medias homogéneas. Finalmente, C 30 en seca, Río en seca y C 16 en lluvia y seca no presentaron diferencias significativas entre sí y presentaron las abundancias medias más bajas. En la Planicie también hubo interacción. Al igual que en el Veril Superior, Boya Roja, en lluvia, presentó una media significativamente superior al resto.

Finalmente en Echinometra, los sitios fueron disímiles, no así las épocas y no existió interacción. C16 y Boya Roja no diferían entre sí. C 30 presentó diferencias importantes con el resto y lo mismo ocurrió con el Río, que presentó una media significativamente más baja.

\section{Thalassoma bifasciatum}

Esta especie también es más abundante en los sitios de aguas más limpias y hacia biotopos profundos y con mayor complejidad topográfica como Pendiente y Veril Superior. Boya Roja 
CUADRO 2

Resultados del análisis de varianza y la prueba SNK por biotopos para Halichoeres bivittatus entre épocas del año y los sitios estudiados

TABLE 2

Halichoeres bivittatus ANOVA and SNK tests results by site type between seasons and sites

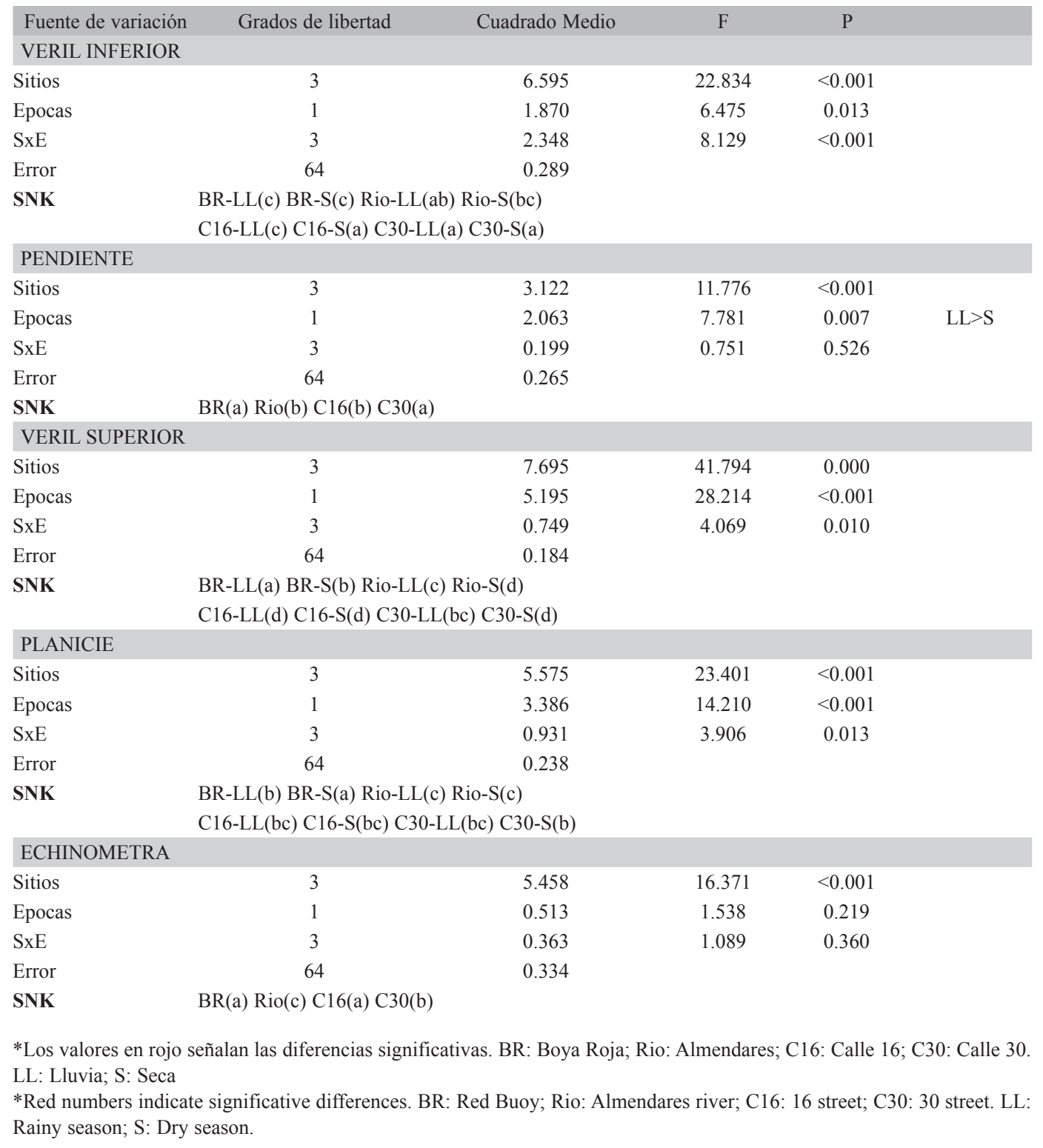


y Río se comportan de forma similar pero en el Veril Inferior de estos dos sitios hay escasez de estos peces, contrario a lo que pasa en los sitios de referencia (Fig. 4). Al analizar los datos por biotopos se obtuvieron los resultados que se exponen a continuación (Cuadro 3).

En el Veril Inferior, se encontraron diferencias significativas entre épocas y entre sitios. La especie es más abundante en la época de lluvia. C 30 y C 16 no diferían entre sí y ambas eran significativamente mayores que Boya Roja y Río, las cuales no diferían entre ellas.

En la Pendiente sí hubo interacción. C 30 en lluvia tuvo una media significativamente más elevada que el resto. En las restantes combinaciones de sitio y época del año la prueba de comparaciones múltiples no arrojó un resultado concluyente y por lo tanto hay que considerar un grupo homogéneo.

Para el Veril Superior, la interacción resultó significativa. La prueba SNK permitió definir que en Boya Roja en la época de seca la abundancia media fue sensiblemente más baja que en el resto de las combinaciones de sitio y época. No fue posible definir diferencias significativas entre otros grupos de medias.

En la Planicie se observaron diferencias significativas sólo entre las épocas del año en las cuales seca resultó presentar una abundancia mayor. Por último en el biotopo Echinometra hubo interacción, pero la prueba de comparaciones múltiples no permitió definir diferencias entre grupos de medias.

Variación en la composición por tallas en sitios con diferente grado de contaminación: En C 30 y C 16 abundan los individuos de Stegastes partitus de $0-3$ y $3-6 \mathrm{~cm}$ siendo la C 16 el sitio con mayor abundancia para los individuos de esta especie. En la lluvia puede observarse como en C 16 hay predominio de adultos, mientras que en seca, la relación entre adultos y juveniles es equivalente. Esta especie aparece

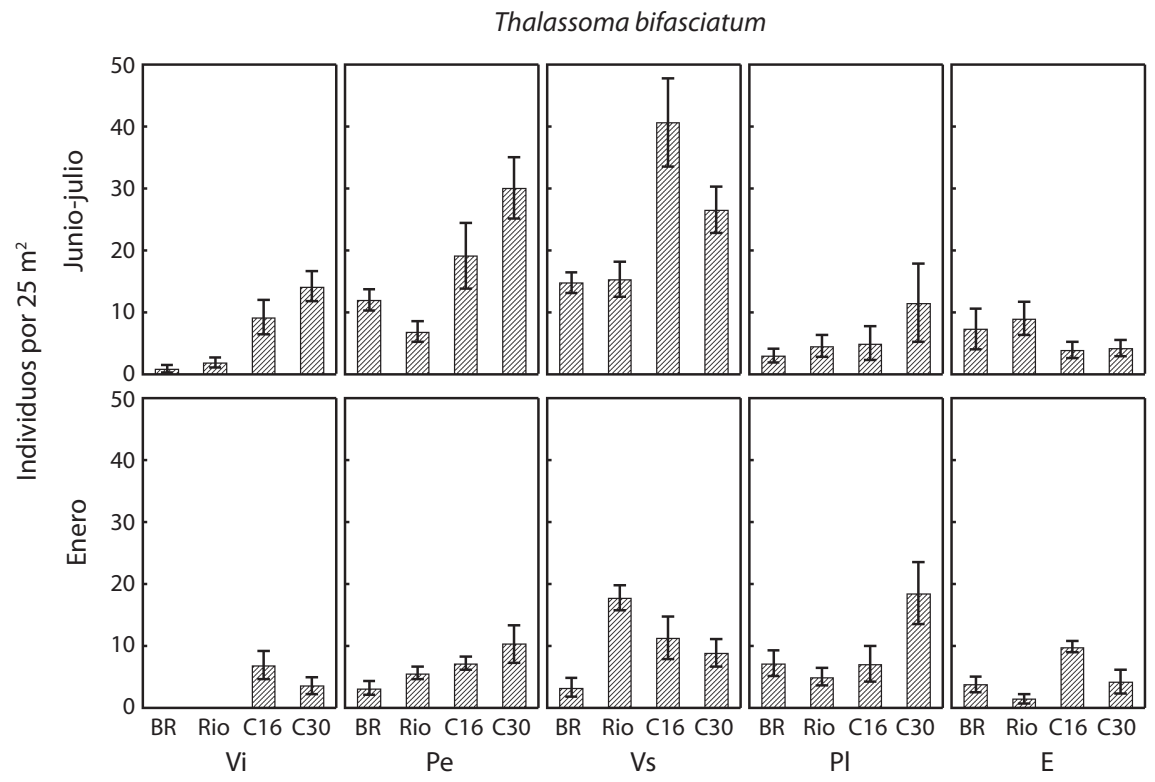

Fig. 4. Abundancia de Thalassoma bifasciatum en los distintos biotopos (E: Echinometra; Pl: Plano Rocoso; Vs: Veril Superior; Pe: Pendiente; Vi: Veril Inferior) para todos los sitios muestreados (BR: Boya Roja; Rio: Almendares; C16: Calle 16; C30: Calle 30). Se representa la media y el error estándar.

Fig. 4. Thalassoma bifasciatum abundance at different sites (E: Echinometra; Pl: Rocky Plain; Vs: Superior Wall; Pe: Wall; Vi: Inferior Wall) in sampled sites (BR: Red Buoy; Rio: Almendares river; C16: 16 street; C30: 30 street). Mean and standard error are represented. 
con tallas mayores (de 6-10cm) en Boya Roja $\mathrm{y}$ en el Río, aunque se encuentra en menor abundancia que en los sitios más limpios donde estas longitudes no son observadas. En Boya Roja son especialmente abundantes los peces grandes en seca, de hecho, son la mayoría de los peces que se encuentran; sin embargo, en lluvia esta relación se desplaza hacia peces más chicos y los grandes desaparecen. Lo contrario ocurre en el Río donde encontramos que los peces mayores son más escasos y aparecen en lluvia aunque la mayor abundancia para este sitio es en seca con juveniles (Fig. 5).

Halichoeres bivittatus presenta abundancia de individuos de todas las tallas en Boya Roja en ambas épocas del año, especialmente en la Planicie y en el Veril. En seca la abundancia de los peces grandes aumenta. El Río presenta pocos individuos de esta especie. En lluvia hay una mayor cantidad de adultos, los cuales son remplazados por juveniles en seca manteniendo una cantidad total de peces similar entre las dos épocas del año. En la C 16 se observan individuos de todas las tallas durante todo el año al igual que los otros dos sitios. Finalmente C 30 también tiene peces de todas las tallas siempre. En el período de seca se observa un aumento de la abundancia de los peces de $3-6 \mathrm{~cm}$ de longitud (Fig. 6).

Para Thalassoma bifasciatum, en seca las tallas se comportan de forma similar entre sitios existiendo una abundancia un tanto mayor en $\mathrm{C}$ 30 y C 16, dado especialmente por juveniles. En lluvia la abundancia se eleva, sobre todo los peces pequeños (de $3-6 \mathrm{~cm}$ y $0-3 \mathrm{~cm}$, respectivamente). Sin embargo, en Boya Roja y el Río los individuos grandes de esta especie disminuyen, fundamentalmente en el Río donde se hacen casi cero (Fig. 7).

\section{DISCUSIÓN}

Fueron tomados en cuenta tres factores posibles de influencia sobre la composición de las asociaciones de peces a lo largo de la costa: el biotopo, la estación del año y la contaminación del agua. El biotopo es determinante en la

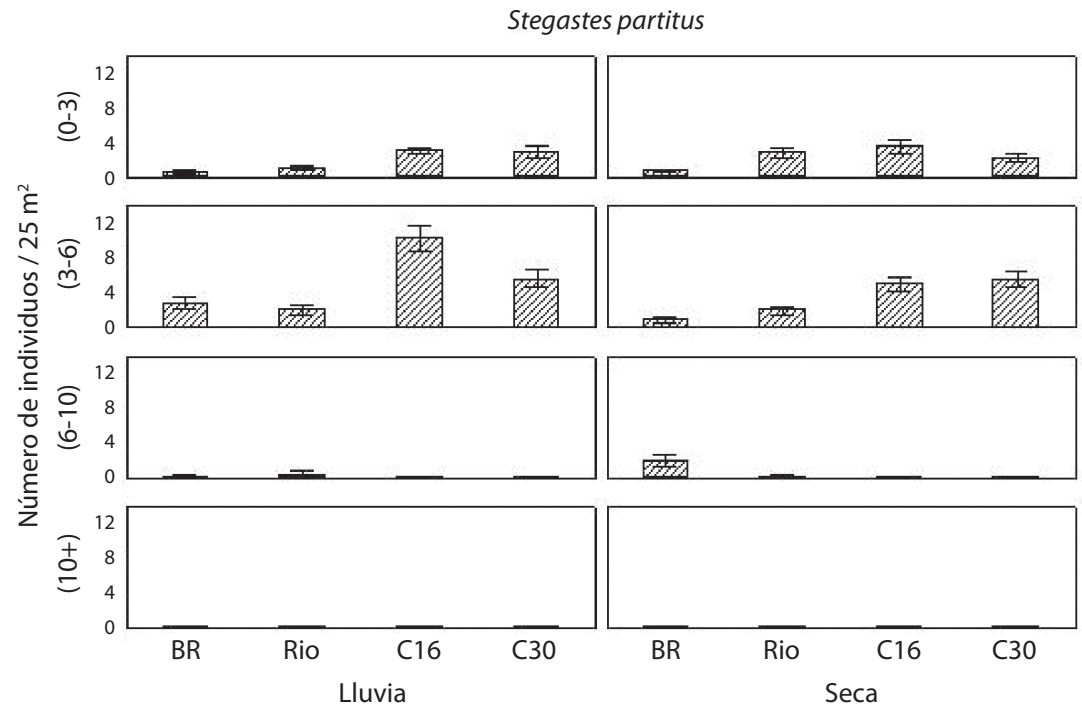

Fig. 5. Abundancia y ámbitos de tallas (longitud en $\mathrm{cm}$ ) de Stegastes partitus en los sitios muestreados (BR: Boya Roja; Rio: Almendares; C16: Calle 16; C30: Calle 30). Se representa la media y el error estándar.

Fig. 5. Stegastes partitus size ranges (length in $\mathrm{cm}$ ) and abundance at sampled sites (BR: Red Buoy; Rio: Almendares river; C16: 16 street; C30: 30 street). Mean and standard error are represented. 


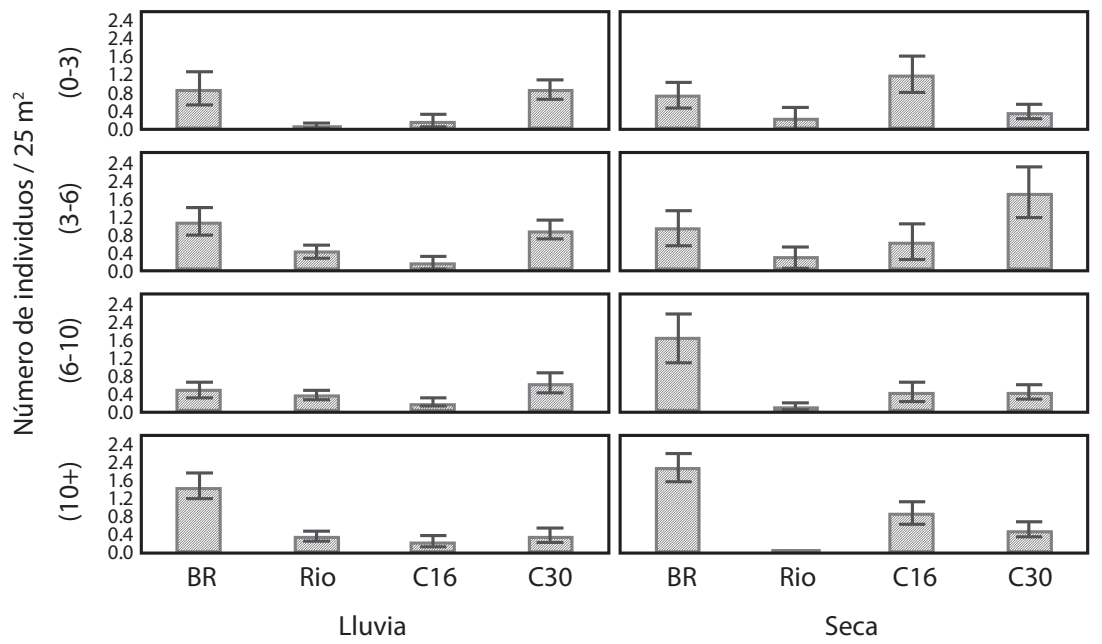

Fig. 6. Abundancia y ámbitos de tallas de Halichoeres bivittatus en los sitios muestreados (BR: Boya Roja; Rio: Almendares; C16: Calle 16; C30: Calle 30). Se representa la media y el error estándar.

Fig. 6. Halichoeres bivittatus size ranges (length in $\mathrm{cm}$ ) and abundance at sampled sites (BR: Red Buoy; Rio: Almendares river; C16: 16 street; C30: 30 street). Mean and standard error are represented.

Thalassoma bifasciatum

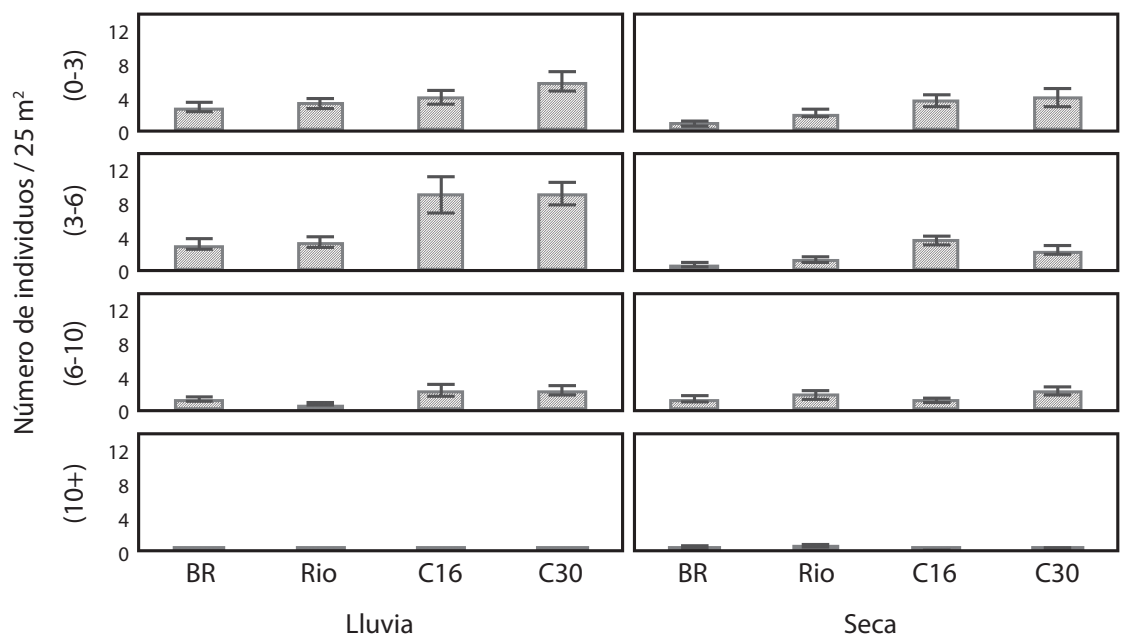

Fig. 7. Abundancia y ámbitos de tallas de Thalassoma bifasciatum en los sitios muestreados (BR: Boya Roja; Rio: Almendares; C16: Calle 16; C30: Calle 30). Se representa la media y el error estándar.

Fig. 7. Thalassoma bifasciatum size ranges (length in $\mathrm{cm}$ ) and abundance at sampled sites (BR: Red Buoy; Rio: Almendares river; C16: 16 street; C30: 30 street). Mean and standard error are represented. 
abundancia y composición de las comunidades de peces. Los principales factores naturales que influyen son el movimiento del agua y la complejidad del sustrato (Bell \& Galzin 1984, Grigg 1994, Chabanet et al. 1997, Lirman 1999) y todos ellos se relacionan con la profundidad, lo que se refleja en la zonación del fondo como bandas paralelas a lo largo de la costa (Nuñez-Lara \& Arias González 1998).

La época del año resultó ser el factor menos influyente de los tres sitios, en concordancia con lo planteado por Aguilar et al. (en prensa). En general la abundancia de los peces tiende a ser mayor en lluvia y se plantea que puede ser debido a que estos meses corresponden con la época de aguas más cálidas y tranquilas, lo cual es propicio para un crecimiento mayor de algas (Guardia 2000a) y asentamiento de larvas y juveniles (Booth \& Beretta 1994).

El aumento de la abundancia de la ictiofauna se debe, en gran medida, al aumento de los juveniles en las poblaciones de peces. Los máximos de reclutamiento que ocurren en los meses de junio-julio pueden explicar los cambios temporales en el número de individuos de las comunidades de peces (Galzin 1987, Letourneur 1996, Pérez-Fleitas 2002). Aguilar \& González-Sansón (1998), observaron que estos máximos se alcanzan rápidamente y a partir de ahí el número de juveniles disminuye también rápido, lo cual provoca que a finales del verano y en los meses de invierno el número de juveniles decrezca notablemente. Esto es evidente en el caso de Thalassoma bifasciatum, que tiene su máximo de abundancia de juveniles en junio y en julio. No obstante, este máximo es inferior en los sitios de aguas contaminadas con respecto a los encontrados en aguas limpias, lo cual se evidencia también en Stegastes partitus. Muchos reclutas arriban a nuevas zonas arrastrados por las corrientes. Su asentamiento depende en gran medida de que el nuevo sitio tenga condiciones propicias. Considerando que la mayor diferencia entre los sitios estudiados está dada por la presencia de contaminantes en el agua, es posible considerar que en estos casos, la contaminación esté influenciando de manera negativa el reclutamiento.
Se han observado cambios en las comunidades bentónicas de la zona que evidencian una influencia de la contaminación. En la zona costera de la desembocadura del Almendares se observaron grandes cantidades de Ulva sp. y Enteromorpha sp., ambas especies indicadoras de contaminación (Aguilar et al. 2000). Así mismo existen variaciones en la composición por especies para las esponjas. Hacia la zona del Río Almendares y la Bahía abundan las esponjas Clathria venosa, Scopalina sp., las cuales se han encontrado muy abundantes en zonas afectadas por la contaminación orgánica mientras que en las estaciones limpias hallamos los géneros de Callyspongia y Aplysina que se consideran indicadora de lugares limpios (Alcolado \& Herrera 1987). Además, la descarga del río y bahía tienen un efecto evidente sobre el cubrimiento y abundancia de algas y corales. Se observa la tendencia a incrementar el cubrimiento por algas en la zonas más influenciadas por el Río y la bahía y el efecto inverso sobre el cubrimiento de corales, este se incrementa en las estaciones de C 30 y C 16 (Aguilar et al. 2000). Esta composición diferencial es un indicador del gradiente ambiental relacionado con la salida de aguas contaminadas del Río Almendares y la bahía, el cual se suaviza hacia las estaciones de C 30 y C 16 donde los índices ecológicos indican condiciones no tan severas (Guardia \& González- Sansón 2000b).

Cuando se trata de realizar un análisis comparativo del tipo de contaminación predominante en las zonas bajo la influencia del Río Almendares y la bahía de La Habana respectivamente no es posible arribar a conclusiones definitivas. Son muy escasos los trabajos sobre las características del agua en la zona costera de Ciudad de La Habana. Morales et al. (1995) muestran gráficos de la distribución horizontal de varios factores en la zona costera que se extiende desde el Río Almendares hasta la bahía de La Habana. En dichos gráficos se aprecia que el oxígeno presenta concentraciones más bajas cerca de la entrada del puerto, lo que se atribuye a la descarga de gran cantidad de materia orgánica. Esta tendencia se observa también en el $\mathrm{pH}$, lo que se explica por la 
producción de ácido sulfhídrico durante la descomposición de la materia orgánica. Por otro lado, las concentraciones de nitritos y nitratos son más altas al este del río debido a la descarga de aguas nitrogenadas por escurrimiento costero. El amonio es más abundante cerca del río y de la bahía. Todo lo anterior es evidencia, principalmente de un proceso de eutrofización, situación que ha sido documentada también por Areces \& Toledo (1985).

Resulta importante señalar que la baja representación de las dos especies más abundantes (Stegastes partitus y Thalassoma bifasciatum) en el Río y la Boya Roja es una evidencia de efectos estresantes. La causa más probable de la disminución de la abundancia de Stegastes partitus en la Pendiente del Río en época de lluvia es el incremento de la descarga de esa corriente en esa época. Ambas especies estuvieron siempre en mayor abundancia hacia los sitios más limpios y biotopos más profundos (Veril Superior-Veril Inferior). Esto concuerda con lo reportado por Aguilar et al. (1997), González-Sansón et al. (1997).

Se observó una tendencia interesante en la especie Stegastes partitus, de aparecer en menor número en zonas de aguas contaminadas. Sin embargo, los ejemplares que aparecían eran de mayor tamaño. Este incremento en el largo de los individuos en las zonas más contaminadas (Boya Roja y Río) pudiera explicarse por la alta eutrofización de las aguas (Areces \& Toledo 1985, Aguilar \& González-Sansón 2000) y por tanto, una mayor disponibilidad de alimento debido a que esta especie se alimenta fundamentalmente de fitobentos. La poca abundancia puede deberse a que estos peces necesitan un sustrato duro y rocoso para hacer sus nidos. Además, según Hernández-Lazo (2001), el tamaño relativo de las gónadas de las hembras y machos de esta especie disminuye a medida que se acercan a la Bahía de manera inversa con el tamaño del cuerpo. Esto podría ser provocado por la contaminación de las aguas. Varios autores han encontrado un decrecimiento del tamaño de las gónadas en experimentos realizados para determinar el efecto de distintos contaminantes sobre los peces (Tulasi et al. 1989, Munkittrick et al. 1992; Choudhury et al. 1993). En general, el impacto de la contaminación a largo plazo trae consigo un decrecimiento en la fecundidad y en la supervivencia de los peces (Kime 1995, Wendelaar 1997).

En el caso de Halichoeres bivittatus, se observó un comportamiento contrario a las otras dos especies estudiadas. Presentó máximos de abundancia en el Veril Inferior y Echinometra debido a que estos individuos se reproducen en aguas someras, pero su reclutamiento tiene lugar en zonas más profundas. Resultó ser más abundante en Boya Roja, especialmente en el Veril Superior y es de suponer que allí se presentan las condiciones para cualquier estadio del ciclo de vida de estos peces. Lo anterior concuerda con lo reportado por González-Sansón \& Aguilar (2002), que plantean que esta especie puede ser más resistente a la sedimentación y a efectos estresantes, que las otras, lo que justificaría su presencia en este sitio (Baelde 1990, Thomas \& Logan 1992, Aguilar et al. 1997, González-Sansón et al. 1997, Aguilar et al. en prensa). Esta especie se encuentra en abundancia en casi cualquier lugar, ya sea arrecife, arenazo, seibadal, manglar. Esto evidencia una amplia adaptabilidad y resistencia de la especie ante el medio. Se recomienda realizar investigaciones posteriores para confirmar una tolerancia mayor de Halichoeres bivittatus hacia agentes estresantes y definir los límites de esta tolerancia. Ya que la misma no es abundante en el sitio cercano al Río, esto podría sugerir que las descargas de este sean demasiado hostiles.

En resumen, la contaminación es un factor influyente en la distribución de las especies de peces. Los sitios de referencia poseen una abundancia mayor que los de aguas más contaminadas. La baja representación de las dos especies más abundantes (Stegastes partitus y Thalassoma bifasciatum) en el Río y la Boya Roja es una evidencia de agentes estresantes. Los representantes de Stegastes partitus fueron más grandes hacia las zonas de aguas contaminadas. Estos peces grandes aparecen en una frecuencia menor, probablemente con gónadas 
menos desarrolladas por efecto de la contaminación. Un comportamiento contrario al resto de las especies estudiadas lo tuvo Halichoeres bivittatus, ya que fue más abundante en Boya Roja. Se plantea que esta especie puede ser más resistente que las otras y se recomiendan estudios posteriores.

\section{RESUMEN}

Esta investigación establece la variación de la abundancia de tres especies de peces seleccionadas en cuatro sitios con diferente grado de contaminación: la Calle 30 y la Calle 16 de Miramar, la Boya Roja de la Bahía de La Habana y la desembocadura del río Almendares. Los conteos fueron realizados desde junio-julio del 2003 hasta enero del 2004. Los sitios de trabajo fueron divididos en cinco biotopos según las características del fondo. El método empleado fue el censo visual en un cuadrado de $5 \mathrm{~m}$ de lado. En cada uno se hicieron nueve conteos cada vez. Se contaron todos los individuos de las especies Stegastes partitus, Thalassoma bifasciatum y Halichoeres bivittatus. Las especies fueron más abundantes en zonas de aguas más limpias, excepto Halichoeres bivittatus que parece presentar mayor resistencia a zonas con cierta contaminación.

Palabras clave: Stegastes partitus, Halichoeres bivittatus, Thalassoma bifasciatum, peces, abundancia espacial.

\section{REFERENCIAS}

Aguilar, C. 1981. Estudio de la estructura de las comunidades bentónicas en una zona del sublitoral del Norte de La Habana. Trabajo de Diploma, Centro de Investigaciones Marinas, Universidad de La Habana, Habana, Cuba.

Aguilar, C., G. González-Sansón, J. Angulo \& C. Gándara. 1997. Variación espacial y estacional de la ictiofauna en un arrecife de coral costero de la región noroccidental de Cuba. I: Abundancia total. Rev. Invest. Mar. 183: 223-232.

Aguilar, C \& G. González-Sansón. 1998. Variación estacional de la abundancia de juveniles de peces en una zona del sublitoral rocoso de La Habana, Cuba. Rev. Invest. Mar. 19: 38-45.

Aguilar, C \& G. González-Sansón. 2000. Influencia de la contaminación de la Bahía de La Habana, Cuba sobre las asociaciones de peces costeros, 1. Abundancia y Diversidad. Rev. Invest. Mar. 211: 60-70.

Aguilar, C., G. González-Sansón, E. de la Guardia, A. M. Suárez \& J. Trilles. 2000. Inventario de los componentes más comunes de la flora y la fauna del arrecife de coral costero de la caleta de San Lázaro, región noroccidental de Cuba, en el período de 1996 a 1998. Rev. Invest. Mar. 211: 53-60.

Aguilar, C \& G. González-Sansón. 2002. Ecología de la ictiofauna costera alrededor de la desembocadura del río Almendares La Habana, Cuba. Distribución espacial de la abundancia y la diversidad. Rev. Invest. Mar. 231: 5-14.

Aguilar, C., G. González-Sansón, K.R. Munkittrick \& D.L. MacLatchy. en prensa. Fish assemblages on fringe coral reefs of the northern coast of Cuba near Havana Harbor. Ecotox. Env. Saf.

Alcolado, P. \& A. Herrera. 1987. Efectos de la contaminación sobre las comunidades de esponjas en el litoral de La Habana, Cuba. Reporte de Investigación 68, Instituto de Oceanología, Ciudad de La Habana, Cuba.

Areces, J. A. \& L. Toledo. 1985. Características tróficas de la Bahía de La Habana durante el período de seca. Reporte de Investigación 40, Instituto de Oceanología, Ciudad de La Habana, Cuba.

Baelde, P. 1990. Differences in the structure of fish assemblages in Thalassia testudinum beds in Guadeloupe, French West Indies and their ecological significance. Mar. Biol. 105: 163-173.

Bell, J. D. \& R. Galzin. 1984. Influence of live coral cover on coral reefs fish communities. Mar. Ecol. Prog. Ser. 15: $265-274$

Booth, D. J. \& Beretta 1994. Seasonal recruitment, habitat associations and survival of a pomacentrid reef fish in US Virgin Islands. Coral Reefs 13: 81-89.

Chabanet, P., H. Ralambondrainy, M. Amanieu, G. Faure \& R. Galzin. 1997. Relationships between coral reefs substrata and fish. Coral Reefs 16:93-102.

Choudhury, C., A. Ray \& S. Bhattacharya. 1993. Non lethal concentrations of pesticide impair ovarian function in the freshwater perch, Anabas testudineus. Environ. Biol. Fish. 36: 319-324.

Díaz, G. S. 1997. Contribución al análisis de los paisajes del parque metropolitano de La Habana y como base para el ordenamiento territorial. Tesis de Maestría. Facultad de Geografía, Universidad de La Habana, Cuba.

Elliot, J.M. 1971. Some methods for the statistical analysis of samples of benthic invertebrates. Freshwater Biol. Assoc. Sci. Pub. 25: 144. 
Emery, R. A. 1973. Comparative ecology and functional osteology of 14 species of damselfish. Bull. Mar. Sci. 23: $649-770$.

Fausch, K. D., J. Lyons, J.R. Karr \& P.L. Argermeier. 1990. Fish communities as indicators of enviromental degradation. Am. Fish. Soc. Symp. 8: 123-144.

Galzin, R. 1987. Structure of fish communities on French Polynesian coral reef. Mar. Ecol. Prog. Ser. 41: 137145.

González-Sansón, G., C. Aguilar, J. Angulo \& C. Gándara. 1997. Variación espacial y estacional de la ictiofauna en un arrecife de coral costero de la región noroccidental de Cuba II: Diversidad. Rev. Invest. Mar. 183: 233-240.

González-Sansón, G. \& C. Aguilar. 2000. Influencia de la contaminación de la Bahía de La Habana Cuba sobre las asociaciones de peces costero, 2. Análisis multidimensional. Rev. Invest. Mar. 211:71-80.

González-Sansón, G. \& C. Aguilar. 2002. Ecología de la ictiofauna costera alrededor de la desembocadura del río Almendares, La Habana, Cuba. II. Análisis multidimensional. Rev. Invest. Mar. 231:15-26.

González-Sansón, G. \& C. Aguilar. en prensa. Spatial and temporal variations in the abundance of dominant coral reef fish species off the coast of Havana city, Cuba. Rev. Invest. Mar.

Grigg, R. W. 1994. Effects of seawage discharge, fishing pressure and habitat complexity on coral ecosystems and reef fishes in Hawaii. Mar. Ecol. Prog. Ser. 103: 25-34.

Guardia, E. de la. 2000a. Asociaciones de corales, gorgonias y esponjas del sublitoral habanero al Oeste de la Bahía de La Habana. I. Gradiente ambiental. Rev. Invest. Mar. 211-3:1-8.

Guardia, E. de la. 2000b. Evento de blanqueamiento en un arrecife de coral en la costa de La Habana, Cuba. Rev. Invest. Mar. 211:108-110.

Guardia, E. de la \& G. González-Sansón. 2000a. Asociaciones de corales, gorgonias y esponjas del sublitoral habanero al oeste de la Bahía de La Habana, 1. Gradiente ambiental. Rev. Invest. Mar. 211:1-8.

Guardia, E. de la \& G. González-Sansón. 2000b. Asociaciones de corales, gorgonias y esponjas del sublitoral habanero al oeste de la Bahía de La Habana. 2. Índices ecológicos. Rev. Invest. Mar. 211: 9-16.

Hernández-Hernández, I., C. Aguilar \& G. GonzálezSansón. 2006. Variación estacional de la abundancia de especies de peces seleccionadas en el sublitoral rocoso de Ciudad de La Habana, Cuba. Rev. Invest. Mar. 271: 61-68.

Hernández-Lazo, A.T. 2001. Índices morfométricos y organosomáticos de la especie Stegastes partitus en zonas arrecifales del sublitoral habanero con diferente grado de contaminación. Trabajo de Diploma. Centro de Investigaciones Marinas, Universidad de La Habana, Cuba.

Herrera, A. \& P. Alcolado. 1983. Efecto de la contaminación sobre las comunidades de gorgonáceos al Oeste de la Bahía de La Habana. Cienc. Biol. 10: 69-85.

Herrera, A. \& N. Martínez-Estalella. 1987. Efectos de la contaminación sobre las comunidades de corales escleractíneos al Oeste de la Bahía de La Habana. Reporte de Investigación 62, Instituto de Oceanología, Ciudad de La Habana, Cuba.

Ibarra, M. E., A. M. Suárez \& M. Ortiz. 1983. La protección del medio y la utilización racional de los recursos naturales. Rev. Invest. Mar. 4: 125-150.

Karr, J. R. \& D. R. Dudley. 1981. Ecological perspective on water quality goals. Environ. Manag. 5: 55-68.

Kime, D. E. 1995. The effects of pollutions on reproduction in fish. Rev. Fish. Biol. Fish. 5: 52-96.

Letourneur, I. 1996. Dynamics of fish communities on Reunion fringing reefs, Indian Ocean. II. Patterns of temporal fluctuations. J. Exp. Mar. Biol. Ecol. 195: 31-52.

Lirman, D. 1999. Reef fish communities associated with Acropora palmata: relationships to benthic attributes. Bull. Mar. Sci. 65: 235-252.

Milliar, M. I. 1997. Diagnóstico ambiental de los paisajes de la Ciudad de La Habana. Trabajo de Diploma. Facultad de Geografía, Universidad de La Habana, Cuba.

Morales, A., H. Rondón, D. López, G. Díaz, L. Rubiera \& A. Cervato. 1995. Estudio de las causa que originan las penetraciones del mar en el malecón Habanero y principales características hidroquímicas y dinámicas de las aguas del litoral. Informe Científico Técnico, GEOCUBA, Ciudad de La Habana, Cuba. 
Munkittrick, K., M. McMaster, C. Prott, G. Van Der Kraak, I. Smith \& D. Dixon. 1992. Changes in maturity, plasma sex steroid levels, hepatic mixed function oxygenase activity and the presence of external lesions in lake white fish exposed to bleached kraft mill effluent. Canad. J. Fish Aqua. Sci. 49: 1560-1569.

Nuñez-Lara, E. \& E. Arias-González. 1998. Relationship between reef fish community structure and enviromental variables in the southern Mexican Caribbean. Fish Biol. 53: 209-221.

Pérez-Fleitas, E. 2002. Estructura de las comunidades de peces del arrecife coralino del Rincón de Guanabo. Trabajo de Diploma. Centro de Investigaciones Marinas, Universidad de La Habana, Cuba.

Thomas, M. L. \& A. Logan 1992. A guide to the ecology of shore line and shallow water marine communities of Bermudas. Bermuda Biological Station for Research. 23: $30-43$.
Tulasi, S., P. Reddy \& J. Rao. 1989. Effect of lead on the spawing potential of Anabas testudineus. Bull. Env. Tox. 43: 858-863.

Waldner, R \& D. Robertson. 1980. Patterns of habitat partitioning by eight species of territorial Caribbean damselfish. Bull. Mar. Sci. 30: 171-188.

Wendelaar, S. 1997. The stress response in fish. Physiol. Rev. 773: 591-625.

Zar, J. H. 1996. Biostatistical analysis. Prentice Hall, Nueva Jersey, EEUU.

\section{REFERENCIA DE INTERNET}

Proyecto Cub- 80- 001. Investigación y control de la contaminación marina. PNUMA -PNUD-UNESCO. (Consultado: 20 de noviembre 2008, www.seaweb. org/background/abstracts/fisheries/2001/01) 